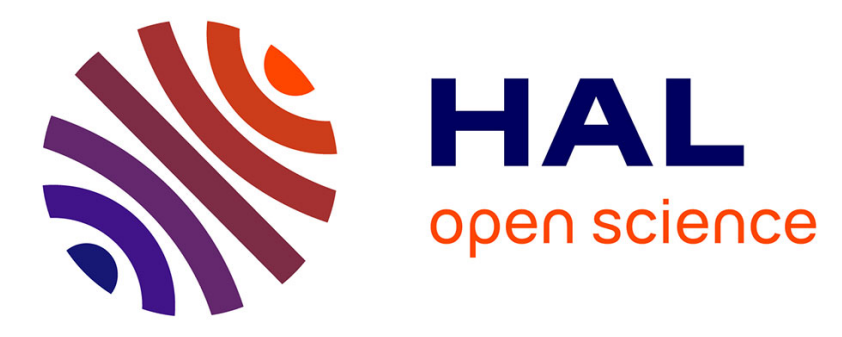

\title{
Feedback control algorithms for the dissipation of traffic waves with autonomous vehicles
}

\author{
Maria Laura Delle Monache, Thibault Liard, Anais Rat, Raphael E. Stern, \\ Rahul Bhadani, Benjamin Seibold, Jonathan Sprinkle, Daniel B. Work, \\ Benedetto Piccoli
}

\section{To cite this version:}

Maria Laura Delle Monache, Thibault Liard, Anais Rat, Raphael E. Stern, Rahul Bhadani, et al.. Feedback control algorithms for the dissipation of traffic waves with autonomous vehicles. Computational Intelligence and Optimization Methods for Control Engineering, Springer, pp.275-299, 2019, 10.1007/978-3-030-25446-9_12 . hal-01930724

\section{HAL Id: hal-01930724 \\ https://hal.inria.fr/hal-01930724}

Submitted on 22 Nov 2018

HAL is a multi-disciplinary open access archive for the deposit and dissemination of scientific research documents, whether they are published or not. The documents may come from teaching and research institutions in France or abroad, or from public or private research centers.
L'archive ouverte pluridisciplinaire HAL, est destinée au dépôt et à la diffusion de documents scientifiques de niveau recherche, publiés ou non, émanant des établissements d'enseignement et de recherche français ou étrangers, des laboratoires publics ou privés. 


\title{
Feedback control algorithms for the dissipation of traffic waves with autonomous vehicles
}

\author{
M. L. Delle Monache*, T. Liard ${ }^{\dagger}$, A. Rat ${ }^{\ddagger}$, R. Stern ${ }^{\S}$, \\ R. Badhani, B.Seibold ${ }^{\|}$, J. Sprinkle ${ }^{* *}$ D. Work ${ }^{\dagger \dagger}$ B. Piccoli ${ }^{\ddagger \dagger}$
}

\begin{abstract}
This article considers the problem of traffic control in which an autonomous vehicle is used to regulate human piloted traffic to dissipate stop and go traffic waves. We first investigate the controllability of well-known microscopic traffic flow models, namely $i$ ) the Bando model (also known as the optimal velocity model), ii) the follow-the-leader model, and iii) a combined optimal velocity follow the leader model. Based on the controllability results, we propose three control strategies for an autonomous vehicle to stabilize the other, human-piloted traffic. We subsequently simulate the control effects on the microscopic models of human drivers in numerical experiments to quantify the potential benefits of the controllers. Based on the simulations, finally we conduct a field experiment with 22 human drivers and a fully autonomous-capable vehicle, to assess the feasibility of autonomous vehicle based traffic control on real human piloted traffic. We show that both in simulation and in the field test that an autonomous vehicle is able to dampen waves generated by 22 cars, and that as a consequence, the total fuel consumption of all vehicles is reduced by up to $20 \%$.
\end{abstract}

\section{Introduction}

Currently, the vehicular transportation system is undergoing a major transition from vehicles in which humans are responsible for all driving tasks, to one in which automation is responsible for all driving tasks. The transition is defined [49] in terms of various levels of automation. The levels range from level one autonomous vehicles (AVs) available today that provide the driver with minor technological assistance (e.g., stability control or lane correction assist), to level five AVs which operate autonomously in all scenarios and in which humans cannot intervene. As the penetration rate of vehicles at each level of automation increases and shifts up the scale, new opportunities are arising to use automated vehicles to begin controlling the overall traffic flow.

A paradigm of traffic control in which some automated vehicles are also acting as traffic control devices is beginning to emerge. In particular, the works $[10,57,16,58,26]$, explore the possibility of adaptive cruise controlled vehicles (e.g., level one automation) to influence traffic flow, for example by smoothing the flow and/or increasing the flow rate. On the extreme end, when the adaptive cruise

${ }^{*}$ Univ. Grenoble Alpes, Inria, CNRS, Grenoble INP, GIPSA-Lab, 38000 Grenoble, France, email: ml.dellemonache@inria.fr ${ }^{\dagger}$ Univ. Grenoble Alpes, Inria, CNRS, Grenoble INP, GIPSA-Lab, 38000 Grenoble, France, email: thibault.liard@inria.fr

${ }^{\ddagger}$ Department of Mathematics, University of Rutgers - Camden, NJ, USA, email: anais.rat@gmail.com

$\S$ Department of Civil and Environmental Engineering \& Institute for Software Integrated Systems, Vanderbilt University, 2301 Vanderbilt Place, Nashville, TN 37235-1826, USA, email: raphael.stern@vanderbilt.edu

๑ Department Electrical and Computer Engineering, University of Arizona, 1230 E. Speedway Blvd., Tucson, AZ 85721-0104 USA, email: rahulbhadani@catworks.arizona.edu

"Department of Mathematics, Temple University, 1805 N. Broad Street, Philadelphia PA 19122, USA, email: seibold@temple.edu

** Department Electrical and Computer Engineering, University of Arizona, 1230 E. Speedway Blvd., Tucson, AZ 85721-0104 USA, email: sprinkle@ece.arizona.edu

${ }^{\dagger}$ Department of Civil and Environmental Engineering \& Institute for Software Integrated Systems, Vanderbilt University, 2301 Vanderbilt Place, Nashville, TN 37235-1826, USA, email: dan.work@vanderbilt.edu

$¥ \ddagger$ Department of Mathematics, University of Rutgers - Camden, NJ, USA, email: piccoli@camden.rutgers.edu 
controlled vehicles are also endowed with communication capabilities between vehicles (referred to as Cooperative Adaptive Cruise Control (CACC) systems), small headways can be achieved and substantial increases in freeway throughput can be obtained [29, 54, 50, 12, 9, 3, 23, 5, 44]. On the experimental side, field experiments with commercial adaptive cruise control vehicles illustrate current technology may in fact amplify traffic oscillations [33], while correctly designed CACC systems dissipate these oscillations [33, 14]. An experiment to harmonize speeds on a US freeway was recently reported [32, 31].

The use of vehicles as traffic controllers may also integrate with more classical traffic control infrastructure, such as ramp meters and variable speed limit systems [35, 19, 51, 20, 43, 38, 15, 37], or systems which combine the two strategies $[18,39,30,17]$. One limitation of the infrastructure based solutions is their limited spatial resolution, as well as the need for driver compliance in the case of speed advisory based systems. The interest to use automated vehicles for traffic control is partly motivated by these limitations.

Considering the new direction of traffic control in which automated vehicles act as actuators, the main contribution of this article is to propose and assess control algorithms designed to dissipate stop and go traffic waves with an autonomous vehicle. Stop and go traffic waves are present on freeways and have many triggering events, such as lane changes. Strikingly, in the seminal experiments of Sugiyama et al. [52] and Tadaki et al. [55], human driving behavior alone was shown to be sufficient to trigger stop and go waves. The experiments were conducted on a single lane circular track with real human drivers, and the uniform flow at the experiment start quickly breaks down into a persistent stop and go wave that travels against the flow of traffic.

Motivated by this experiment, we design control algorithms to be implemented on an autonomous vehicle with the goal of dissipating stop and go waves caused by human driving behavior. We proceed as follows. First we model the vehicular traffic at the microscopic scale using one or a combination of two well-known microscopic models, namely $i$ ) the Bando or optimal velocity (OV) model [1] and the ii) follow the leader (FTL) [48, 47, 42]. With the models defined, we show that traffic described by a linerization of the FTL model is not controllable via a single AV, implying the nonlinear FTL model is not linearly controllable. This result prevents the use of simple linear controllers to stabilize the traffic around the uniform speed equilibrium traffic state. On the other hand, we show that traffic described by the optimal velocity model is locally controllable by an autonomous vehicle. We show that for driving dynamics that include both the optimal velocity and follow the leader terms, the resulting model is also locally controllable ${ }^{1}$

We then proceed to design three different controls in which the AV is used to dampen stop and go waves. The first two are based on Lyapunov functions and only require measurements of the AV speed and the speed of nearby vehicles, while the third is a PID-type control in which the AV is controlled using only measurements of its own speed over several proceeding timesteps.

We first assess the effectiveness of the wave dampening controllers though numerical experiments. In addition to showing a reduction in the wave strength, we also quantify the benefits in terms of a reduction of total fuel consumed by all vehicles in the simulation. Based on the positive performance in simulation, we proceed to field validate one control algorithm with a dive-by-wire autonomous capable vehicle on a track with 22 vehicles driven by humans. The experiment shows that stop and go waves can be dampened, and the projected reduction of fuel consumption from the simulations (approximately $20 \%$ ) is confirmed via real-time fuel consumption loggers installed on the experimental vehicles.

The remainder of the article is organized as follows. In Section 2, we review the main microscopic traffic flow models investigated in this work. Section 3 establishes the main controllability results of the models, while Section 4 describes the design and testing the wave dissipating controllers in simulation. In Section 5 we test one of the wave dampening controllers in a field experiment with real human drivers and an autonomous vehicle. Limitations and future directions are explored in Section 6.

\section{Microscopic traffic models}

Traffic models are usually defined in categories, depending on the scale at which they represent vehicular traffic, including microscopic, mesoscopic, macroscopic and cellular. For a review of models at various scales see [21, 2, 13, 41]. Moreover, some approaches are based on model-agnostic simulation tools, such as deep learning and neural networks [22, 7, 25, 60].

\footnotetext{
${ }^{1}$ Established for $n<=9$ vehicles and conjectured for $n>9$.
} 
In this paper we focus on microscopic models. Microscopic models are suitable for in-silico verification before experimental testing since they describe human driving behavior at the individual vehicle level. One such model is the combined Bando and follow the leader model, which can be formulated as $\dot{x}_{i}=v_{i}$, $\dot{v}_{i}=f\left(x_{i+1}, x_{i}, v_{i+1}, v_{i}\right)$, where $x_{i}$ is the position of $i$-th car, $v_{i}$ its velocity, and $i+1$ is the index of the car ahead.

More precisely, for what concerns the follow the leader model, it was introduced in [48, 47, 42] and it assumes that the acceleration of a vehicle is given by the neighbouring vehicles. The main influence comes from the next vehicle, whose index is $i+1$, that is also called leading vehicle. The main dynamics described by this model is given by:

$$
\left\{\begin{aligned}
\dot{x_{i}} & =v_{i} \\
\dot{v_{i}} & =\frac{v_{i+1}-v_{i}}{x_{i+1}-x_{i}} .
\end{aligned}\right.
$$

This model has the following properties:

- The acceleration depends on the relative velocity $\Delta v=v_{i+1}-v_{i}$

- The velocity $v_{i}(t)$ of the vehicle depends on the velocity of the vehicle in front such that the distance from the vehicle in front is safe.

A drawback of these models is that the acceleration is zero when the relative velocity is zero independently of the headway $d \equiv x_{i+1}-x_{i}$ That is, extremely small headways are allowed even when travelling with extremely high speed, [36]. A model that fixed this problem is the optimal velocity model (introduced by [1]) that describes the adaptation of the actual speed to the optimal velocity $V(\cdot)$ which stands for the desired speed defined by

$$
V(x)=v_{\max } \frac{\tanh \left(x-l_{v}-d_{s}\right)+\tanh \left(l_{v}+d_{s}\right)}{1+\tanh \left(l_{v}+d_{s}\right)},
$$

where $l_{v}$ is the length of cars and $d_{s}>0$ is the safe distance between cars. The optimal velocity has the property that it tends to zero for small headways and it achieves the maximum value for large headways. The full model reads:

$$
\left\{\begin{array}{l}
\dot{x_{i}}=v_{i} \\
\dot{v}_{i}=V\left(x_{i+1}-x_{i}\right)-v_{i} . \quad 1 \leqslant i \leqslant N
\end{array}\right.
$$

In this model, a driver controls acceleration or deceleration according to the difference between the optimal velocity and his own velocity. The equilibrium point for this model is achieved when all cars at constant speed and with the same headway, [27]. For this model it is possible to derive a feedback law such that the controlled traffic system is stable, [34].

\section{Controllability results for microscopic models}

In this Section, we provide theoretical results about controllability for the microscopic models introduced in Section 2. More precisely, we will focus on a ring-road setting, which reproduce the situation of the celebrated Sugiyama experiment [53] with a single AV, which can be controlled, and investigate the controllability of the corresponding control system.

Let us first recall some basic facts about control systems, referring the reader to the books [4] and [8] for details. A control systems is a dynamical system written as:

$$
\dot{y}=f(y, u)
$$

where $y \in \mathbb{R}^{n}$ represents the state of the system and $u \in U \subset \mathbb{R}^{m}$ represents the control vector, i.e. the parameters which can be chosen by an external agent. In our setting $y$ represent the state of the traffic model (e.g. position and velocity of cars) and $u$ the acceleration or speed of the AV which can be controlled.

A system (4) is said controllable if for every states $y_{1}, y_{2}$, there exists $T>0$ and a control function $\bar{u}:[0, T] \rightarrow U$ such that the solution to the Chauchy problem $\dot{y}=f(t, \bar{u}(t)), y(0)=y_{1}$ satisfies $y(T)=y_{2}$. In other words we can steer the system from $y_{1}$ to $y_{2}$ in time $T>0$ with a suitable control $\bar{u}$. Similarly a system is said locally controllable at $\bar{y}$ if for every $\delta>0$ sufficiently small there exists $T>0$ 


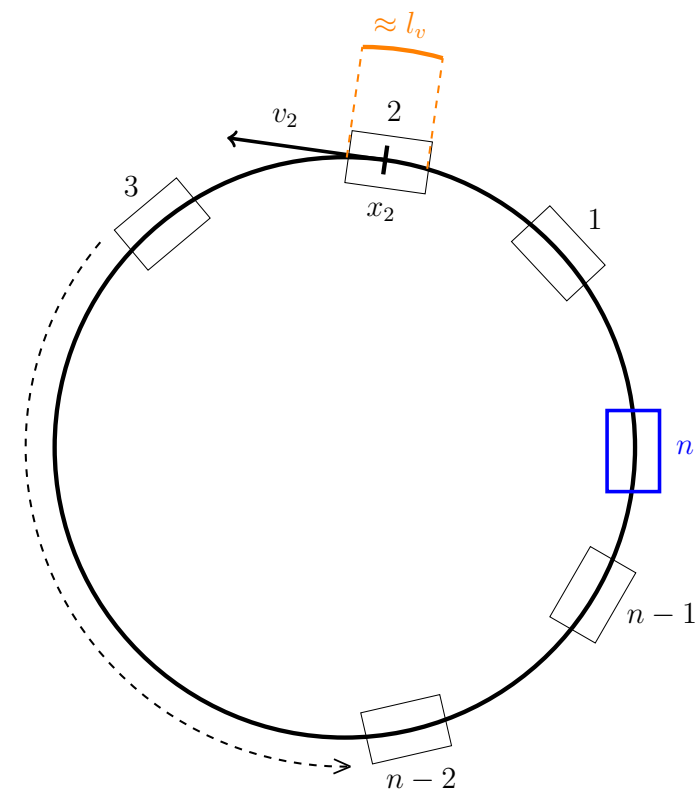

Figure 1: Sketch of $n$ vehicles on a trajectory along a ring of length $L$.

such that we can steer the system from $\bar{y}$ to any $y$ with $|y-\bar{y}|<\delta$ in time $T$. In other words we can reach sufficienly close states in uniformly bounded time.

An important tool to investigate controllability (and local ones) is linearization. Given a control system (4), such that $U$ contains a neighborhood of 0 , and $\bar{y}$, we can consider the linearized system at $\bar{y}$ :

$$
\dot{z}=A \cdot z+B \cdot u
$$

where $A=D_{y} f(\bar{y}, 0)$ (the Jacobian matrix of $f$ w.r.t. $y$ computed at $(\bar{y}, 0)$ ) and $B=D_{u} f(\bar{y}, 0)$ (the Jacobian matrix of $f$ w.r.t. $u$ computed at $(\bar{y}, 0))$. For linear systems, there is a simple criterion for controllability. First, given the linear control system (5), define the Kalman controllability matrix:

$$
K(A, B)=\left[B, A \cdot B, \ldots, A^{n-1} \cdot B\right]
$$

Since $A$ is an $n \times n$ matrix and $B$ is a $n \times m$ matrix, then $K(A, B)$ is an $n \times n \cdot m$ matrix. We can now state the follwing (see [4, Theorem 3.6.2]):

Theorem 1. (Kalman controllability theorem) The system (5) is controllable if and only if the matrix $K(A, B)$ has full rank (i.e. equal to $n$ ).

The fact that we can limit ourselves to the exponent $n-1$ in the definition of $K(A, B)$ follows from Cayley-Hamilton Theorem, which ensures that $A$ is root of its characteristic polynomial, i.e. there exists $\alpha_{i}$ such that $A^{n}=\sum_{A}^{i} i=0^{n-1} \alpha_{i} A^{i}$.

The local controllability of a system can be investigated by looking at its linearization. More precisely we have the following (see [4, Theorem 3.7.1]):

Theorem 2. Consider a control system (4) and its linearization (5) at a given state $\bar{y}$. If (5) is controllable then (4) is locally controllable at $\bar{y}$.

The converse of this Theorem is not true: a nonlinear control system may be controllable even if its linearization fails to be controllable. However, the controllability of the linearization ensures the existence of linear stabilizing feedbacks, i.e. control laws of the type $u(y)=K \cdot y$ that renders the system (4) locally asymptotically stable at $\bar{y}$, see [4, Theorem 4.2 .3$]$.

We are now ready to define our control system for traffic control on a ring-road via one AV, see Figure 1. Let $(a, b) \in \mathbb{R}^{2} \backslash\{(0,0)\}$ and consider the control system of $n$ vehicles along a ring-road of length $L$ 
described by the FTL-Bando model:

$$
\left\{\begin{array}{l}
\dot{x}_{i}=v_{i}, \quad 1 \leqslant i \leqslant n \\
\dot{v}_{i}=a \frac{v_{i+1}-v_{i}}{\left(x_{i+1}-x_{i}\right)^{2}}+b\left[V\left(x_{i+1}-x_{i}\right)-v_{i}\right], \quad 1 \leqslant i \leqslant n-1, \\
\dot{v}_{n}=u
\end{array}\right.
$$

where $x_{i}$ is the position of the $i^{\text {th }}$ vehicle, $v_{i}$ its velocity and $V(\cdot)$ stands for the desired speed which is defined in 2. Notice that we can control only the acceleration $u$ of the $n$-th vehicle, which represents the AV.

Remark 1. If $b=0$, the nonlinear control system (7) is the Follow-The-Leader model (FTL). If $a=0$, the system (7) is the optimal velocity model.

Our aim is to steer the system, (7) to a speed equilibrium state, i.e. a state so that all vehicles have the same velocity $\bar{v}$. If $b \neq 0$, i.e. if there is a non vanishing optimal velocity term, a speed equilibrium corresponds to all vehicles which are not controlled (i.e., all vehicles but the $n$-th ones) having the same headway distance $d$, while the AV may have a different headway. Moreover, to be in speed equilibrium we must have $V(d)=\bar{v}$, thus $d$ is fixed once $\bar{v}$ is fixed. On the other side if $b=0$ then all states with same speed are an equilibrium with any set of headways. We summarize this analysis in the following Lemma:

Lemma 1. Consider the control system (7) and a fixed speed $\bar{v}$.

If $b \neq 0$ then the following holds. Let $d$ be such that $V(d)=\bar{v}$. If $d \leqslant \frac{L}{n}$ then the only speed equilibrium is given by $x(t)$ such that $x_{i+1}(t)-x_{i}(t) \equiv d, i=1, \ldots, n-1$ and $\dot{x}_{i}(t) \equiv \bar{v}, i=1, \ldots, n$. Otherwise there exists no speed equilibrium.

If $b=0$ then every $x(t)$ such that $\dot{x}_{i}(t) \equiv \bar{v}, i=1, \ldots, n$, is an equilibrium.

Now fix $(d, \bar{v}) \in \mathbb{R}_{+}^{2}$, with $V(d)=\bar{v}$ if $b \neq 0$, then we rewrite (7) as

$$
\dot{Y}=f(Y, u) \text {. }
$$

where $Y=\left(y_{i}\right)_{i=1, \cdots, 2 n-1}=\left(x_{2}-x_{1}-d, \ldots, x_{n}-x_{n-1}-d, v_{2}-v_{1}, \ldots, v_{n}-\bar{v}\right)$ and $f$ is defined by

$$
f(Y, u)=\left(\begin{array}{c}
y_{n} \\
\vdots \\
y_{2 n-2} \\
a\left[\frac{y_{n+1}}{\left(y_{2}+d\right)^{2}}-\frac{y_{n}}{\left(y_{1}+d\right)^{2}}\right]+b\left[V\left(y_{2}+d\right)-V\left(y_{1}+d\right)-y_{n}\right] \\
\vdots \\
a\left[\frac{y_{2 n-2}}{\left(y_{n-1}+d\right)^{2}}-\frac{y_{2 n-3}}{\left(y_{n-2}+d\right)^{2}}\right]+b\left[V\left(y_{n-1}+d\right)-V\left(y_{n-2}+d\right)-y_{2 n-3}\right] \\
u-a \frac{y_{2 n-2}}{\left(y_{n-1}+d\right)^{2}}-b\left[V\left(y_{n-1}+d\right)-\left(y_{2 n-1}-y_{2 n-2}+\bar{v}\right)\right] \\
u
\end{array}\right)
$$

Notice that the vector $Y$ has $2 n-1$ component: $n-1$ differences between the headway of the $n-1$ not controlled vehicles and the equilibrium headway $d, n-1$ velocity differences with leading vehicle for the not controlled vehicles and the difference of AV velocity with the equilibrium ones. Moreover we have the relation $x_{1}-x_{n}=L-\sum_{i=1}^{n-1} y_{i}+(n-1) d$ expressing the headway of the AV. We are interested in speed equilibria with equispaced cars, thus we notice that $Y^{*}=0 \in \mathbb{R}^{2 n-1}$ is the only equilibrium of (8) if $b \neq 0$ and $u=0$. In other words $\left(Y^{*}, 0\right)$ is a speed equilibrium of the controlled system (8), i.e. $f\left(Y^{*}, 0\right)=0$. Notice that for $d=\frac{L}{n}, V(d)=\bar{v}$, at the speed equilibrium $(0,0)$ all vehicles are equispaced and drive at the same speed $v_{i}=\bar{v}$.

We are now ready to state the controllability results for the linearized systems at equilibria. First we have the following:

Theorem 3. Let $b=0, a \neq 0$ and $(d, \bar{v}) \in \mathbb{R}_{+}^{2}$. The linearization of system (8) at the speed equilibrium $\left(Y^{*}, 0\right) \in \mathbb{R}^{2 n}$ is not controllable. 
In other words the linearization FTL model at speed equilibrium is not controllable. This does not prevent the nonlinear system to be controllable, but prevents the use of simple linear controls to drive the system to equilibrium.

Our next result is the following:

Theorem 4. Let $a=0, b \neq 0,(d, \bar{v}) \in \mathbb{R}_{+}^{2}$ with $V(d)=\bar{v}$. The linearization of system (8) at the speed equilibrium $\left(Y^{*}, 0\right) \in \mathbb{R}^{2 n}$ is controllable. Therefore, the nonlinear control system (8) is locally controllable at $\left(Y^{*}, 0\right)$.

Finally for the combined FTL-OV model we have the following:

Theorem 5. We assume that $n \leqslant 9$. Let $(a, b) \neq 0,(d, \bar{v}) \in \mathbb{R}_{+}^{2}$ with $V(d)=\bar{v}$. If $a \neq\left(y^{*}+d\right) V^{\prime}\left(y^{*}+\right.$ d) and $b \neq 0$, the linearization of system (8) at the equilibrium point $\left(Y^{*}, 0\right) \in \mathbb{R}^{2 n}$ is controllable. Therefore, the nonlinear control system (8) is locally controllable at $\left(Y^{*}, 0\right)$.

Remark 2. Since the Optimal Velocity model is locally controllable, one expect the same to be true for the combined FTL-OV model, except possibly some resononant value of the parameters. This is exactly what it proved in Theorem 5. The complexity of the system does not allow to deal with arbitrary large dimensione (only for $n \leqslant 9$ ), but we conjecture that, for $n>9$, the nonlinear control system (8) is still locally controllable at $\left(Y^{*}, 0\right)$.

The proofs of the Theorems are postponed to the Appendix.

\section{Controls and simulations}

In this Section we describe control algorithms to stabilize a FTL-Bando model to a speed equilibrium and test them in silico, i.e. via simulations, on a setting reproducing the Sugiyama experiment [53].

We start defining a Proportional-Integral-Derivative (PID)-type control, which is based on velocity measures of the AV over a fixed time horizon. The control includes saturation terms to avoid collisions and too large headways for the AV. Then we define controls based on Lyapunov functions, using the fact that the system is control affine, see see [8, Definition 3.12], i.e. the control appear linearly with a vector coefficient depending on state. Such techniques are usually referred to as Jurdjevic-Quinn controls [24].

\subsection{PID control}

The idea behind this controller is that the autonomous vehicle may estimate the average speed of the vehicles in front, and then drive according to the average speed, safety permitting. An estimate of the average speed required by the controller is obtained by measuring the autonomous vehicle speed over a large enough time horizon. Note that this requires that there are several waves present, not just a single one, so that the past is informative of the future.

The controller determines a command velocity $u$ following a standard proportional integral control logic. In order for the controller to be efficient it needs to be augmented with saturation: for small gaps the autonomous vehicle should follow the lead vehicle speed to avoid dangerous situations, while for large gaps, the autonomous should catch up to the lead vehicle.

More precisely, this controller estimates the desired velocity, $V_{d}$, as a temporal average of the autonomous vehicle's own velocity over an interval. Letting $v_{1}^{\mathrm{AV}}, \ldots, v_{m}^{\mathrm{AV}}$ denote the autonomous vehicles velocities over the last $m$ measurements, the desired velocity is computed as the temporal average $V_{d}=\frac{1}{m} \sum_{j=1}^{m} v_{j}^{\mathrm{AV}}$. In practice, we choose $m$ corresponding to a 38 second interval, which is approximately the time required to travel one lap around the ring.

The desired average velocity is then translated into a target velocity depending on the current gap between the autonomous vehicle and lead vehicle:

$$
v^{\text {target }}=V_{d}+1 \frac{\mathrm{m}}{\mathrm{s}} \times \min \left(\max \left(\frac{\Delta x-7 \mathrm{~m}}{23 \mathrm{~m}}, 0\right), 1\right)
$$

This allows the autonomous vehicle to drive faster than the average velocity and catch up to the lead vehicle, should it face a big gap, while at lower gaps the target velocity reduces to the average $V_{d}$.

The commanded velocity is updated via:

$$
u_{j+1}=\beta_{j}\left(\alpha_{j} v_{j}^{\text {target }}+\left(1-\alpha_{j}\right) v_{j}^{\text {lead }}\right)+\left(1-\beta_{j}\right) u_{j},
$$


where the subscript $j$ denotes the time step. This rule (11) chooses the new commanded velocity as a weighted average of the prior commanded velocity, the target velocity, and the lead vehicle's velocity. The weights $\alpha_{j}$ and $\beta_{j}$ depend on the gap as follows:

$$
\alpha_{j}=\min \left(\max \left(\frac{\Delta x-\Delta x^{\mathrm{s}}}{\gamma}, 0\right), 1\right)
$$

In (12), the distance $\Delta x^{\mathrm{s}}$ is a safety distance. We have $\alpha_{j}=0$ if $\Delta x \leq \Delta x^{\mathrm{s}}$ and $\alpha_{j}=1$ if $\Delta x \geq \Delta x^{\mathrm{s}}+\gamma$, meaning that for relatively short gaps, only the lead vehicle's velocity matters, while for relatively large gaps, only the target velocity is averaged with the commanded velocity. The parameter $\gamma$ controls the the rate at which $\alpha$ transitions from 0 to 1 , and is set to $\gamma=2 \mathrm{~m}$ in the current implementation. This means that when the gap is short, the autonomous vehicle has the same speed of the lead vehicle, while when the gap is larger the autonomous speed tends towards the target vehicle, which allows the autonomous vehicle to reduce the gap with the lead vehicle. The parameter $\beta_{j}$ determines how rapidly the controller adjusts to new situations (with more rapid adjustments occurring in more safety-critical situations). At its core, this is a PID controller, but with a saturation at small gaps (for safety purposes), and a saturation at large gaps (so that the autonomous vehicle closes gaps).

The safety distance is implemented as $\Delta x^{\mathrm{s}}=\max (2 \mathrm{~s} \times \Delta v, 4 \mathrm{~m})$. The term $2 \mathrm{~s} \times \Delta v$ represents the recommended safe following headway of $2 \mathrm{~s}$, with a lower bound of $4 \mathrm{~m}$.

\subsection{Lyapunov functions and controls}

The system (8) is a control affine system (see [8, Definition 3.12]). More precisely, (8) can be written as

$$
\dot{Y}=f_{a, b}(Y)+u f_{1}(Y)
$$

with $f_{a, b}(Y)$ given by (9) with $u=0$ and and

$$
f_{1}(Y)=\left(\begin{array}{c}
0 \\
\vdots \\
0 \\
1 \\
1
\end{array}\right)
$$

Since we want to steers the system towards the speed equilibrium, we can consider the control Lyapunov function $V_{1}(Y)=v_{n}-\bar{v}=y_{2 n-1}$. Then its derivative along trajectories is given by $\nabla V_{1}$. $\left(f_{a, b}(Y)+u f_{1}(Y)\right)=\mathbf{e}_{2 n-1} \cdot\left(f_{a, b}(Y)+u f_{1}(Y)\right)=u$. To maximize the decrease we can simply choose

$$
u_{1}=-y_{2 n-1}=-\left(v_{n}-\bar{v}\right) \text {. }
$$

Such control is smooth, vanishes at 0 , and globally asymptotically stabilizes to the speed equilibrium.

Remark 3. To have a different gain we may choose the control $u_{\alpha}=-\alpha y_{2 n-1}=-\alpha\left(v_{n}-\bar{v}\right)$ with $\alpha>0$.

Let us now focus on last two components:

$$
\left(\begin{array}{l}
y_{2 n-2} \\
y_{2 n-1}
\end{array}\right)^{\prime}=\left(\begin{array}{c}
u-a \frac{y_{2 n-2}}{\left(y^{*}+d\right)^{2}} \\
u
\end{array}\right)
$$

We introduce the control Lyapunov function:

$$
V_{2}(Y)=\frac{y_{2 n-1}^{2}}{2}+\frac{y_{2 n-2}^{2}}{2}, \quad \text { for every } Y=\left(\begin{array}{c}
y_{2 n-2} \\
y_{2 n-1}
\end{array}\right) \in \mathbb{R}^{2} .
$$

For every $Y \in \mathbb{R}^{2} \backslash\{(0,0)\}$, choosing $u=-\epsilon \operatorname{sign}\left(y_{2 n-1}+y_{2 n-2}\right)$ with $\epsilon>0$, we have, $\nabla V_{2}(Y) \cdot f(Y, u)=$ $\left(y_{2 n-2}+y_{2 n-1}\right) u-a \frac{y_{2 n-2}^{2}}{\left(y^{*}+d\right)^{2}}<0$. Thus, $V_{2}$ is a control Lyapunov function for the control system (14) satisfying the small control property (see [8, definition 12.1]). Using [8, Theorem 12.4]), the control

$$
u_{2}=-\left(y_{2 n-1}+y_{2 n-2}\right)=-\left(v_{n}-\frac{v_{n-1}+\bar{v}}{2}\right)
$$

is smooth, vanishes at $0 \in \mathbb{R}^{2}$ and globally asymptotically stabilizes the control system (14). 


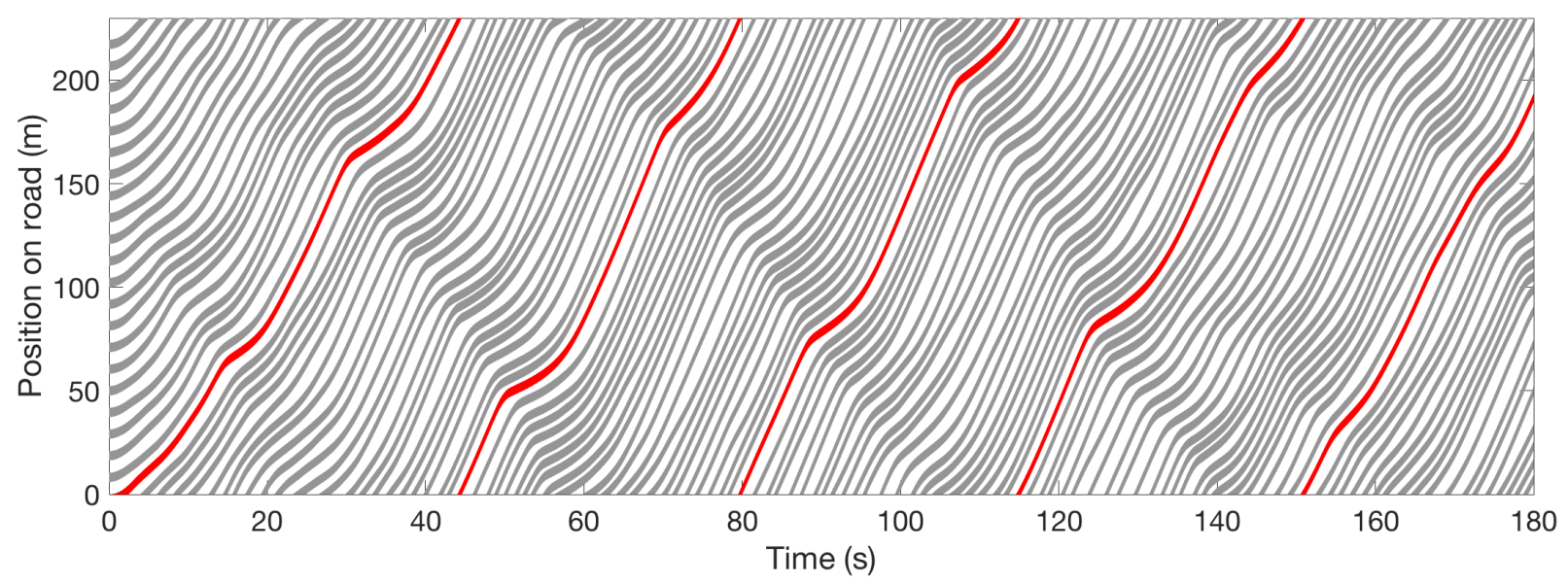

Figure 2: Vehicle trajectories with no control. Note the red trajectory follows a single vehicle which acts identically to all grey trajectories.

\subsection{Simulations}

In this section we demonstrate the capabilities of the control laws described in the previous sections via numerical simulations. The parameters used for the simulations are as follows. We consider $N=22$ vehicles, with one autonomous vehicle and $N=21$ human-driven vehicles. We consider that the humandriven vehicles follow the dynamics described in (7) with the following parameters $a=0.5 \mathrm{~m} / \mathrm{s}^{2}$ and $b=20 \mathrm{~s}^{-1}$. The speed $v_{\max }=9.75 \mathrm{~m} / \mathrm{s}$ and the vehicle length is chosen to be $l_{v}=4.5 \mathrm{~m}$. Such parameters allow to fit the data from the experiment of [53], see Figure 2. In the Figure, the red trajectory corresponds to the autonomous vehicle and the grey ones to human-driven vehicles. One can notice the appearance of strong stop and go waves that start at time 60 s and are propagated on the ring. Visually, there have the effect of "wrinkles" in the pattern.

For comparison, we can now see the effects of the different controls. For the autonomous vehicle we will chose a different dynamics according to the control strategy that we are going to simulate. The simulation begins with all vehicles using the human driving dynamics described in (7) with no control. Control on the AV is activated after $t=40 \mathrm{~s}$.

In Figure 3, we can see the effects of the PID control on vehicle trajectories and on the speed profile in Figure 4. Figure 3 shows that the AV leaves some extra headway to tame the effect of the stop and go waves. Such waves continue to reappear but the control is able to dissipate partly the effects. The velocity profiles, Figure 4, show a strong oscillation reduction for the AV in the time interval [60,100], then oscillations affect the AV and finally are more under control after time $t=160$.

Next we show that stop and go waves can be dampened using the control laws (13) and (15). Since the autonomous vehicle used in the experiment runs with velocity control, we will slightly modify our controls (13) and (15) as follows; let $u_{j} \in \mathbb{R}_{+}$defined by $u_{j}=u\left(t_{j}\right)$ with $t_{j}=j * \Delta t, j \in \mathbb{N}$ and $\Delta t>0$. First, combining (13) with (7), we have $\dot{v}_{n}=-\left(v_{n}-\bar{v}\right)$ with $\bar{v} \in \mathbb{R}_{+}$. Thus, the target velocity is

$$
v_{1, j+1}^{\text {target }}=\left(u_{j}-\bar{v}\right) \exp (-\Delta t)+\bar{v} .
$$

Since $v_{n-1}(s)=v_{n-1}(t):=v_{n-1, j}$ for every $s \in\left[t_{j}, t_{j}+\Delta t\right)$, combining (15) with (7), the target velocity is

$$
v_{2, j+1}^{\mathrm{target}}=\left(u_{j}-\frac{v_{n-1, j}+\bar{v}}{2}\right) \exp (-\Delta t)+\frac{v_{n-1, j}+\bar{v}}{2} .
$$

From now on, $\bar{v}$ is a function depending on time and at every time $t>0, \bar{v}(t)$ is constructed as the minimum of the temporal average speeds of the lead vehicle and the autonomous vehicle over $(0, t)$. More precisely, the target velocity is

$$
\bar{v}_{j}=\min \left(\frac{\sum_{i=1}^{j-1} v_{i}^{\text {lead }}}{j-1}, \frac{\sum_{i=1}^{j-1} u_{i}}{j-1}\right)
$$




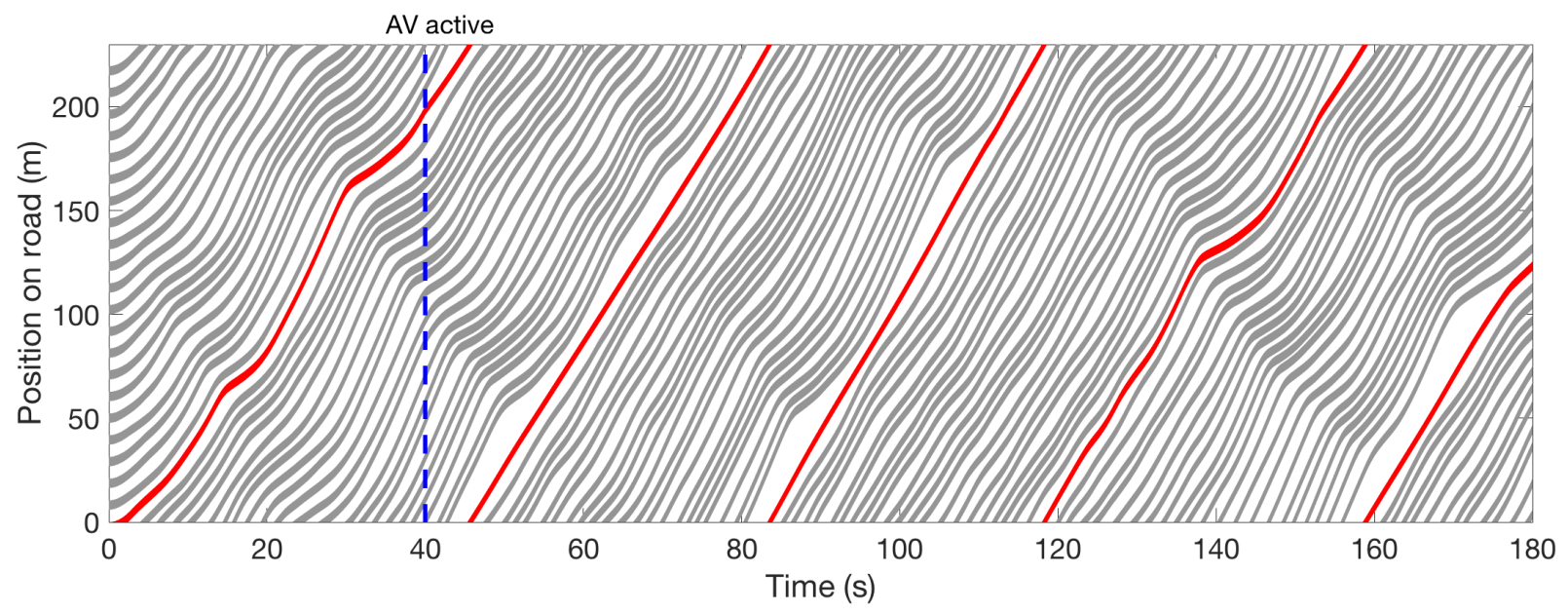

Figure 3: Vehicle trajectories of uncontrolled vehicles (grey) when using the PID control (11) applied only to a single vehicle (red).

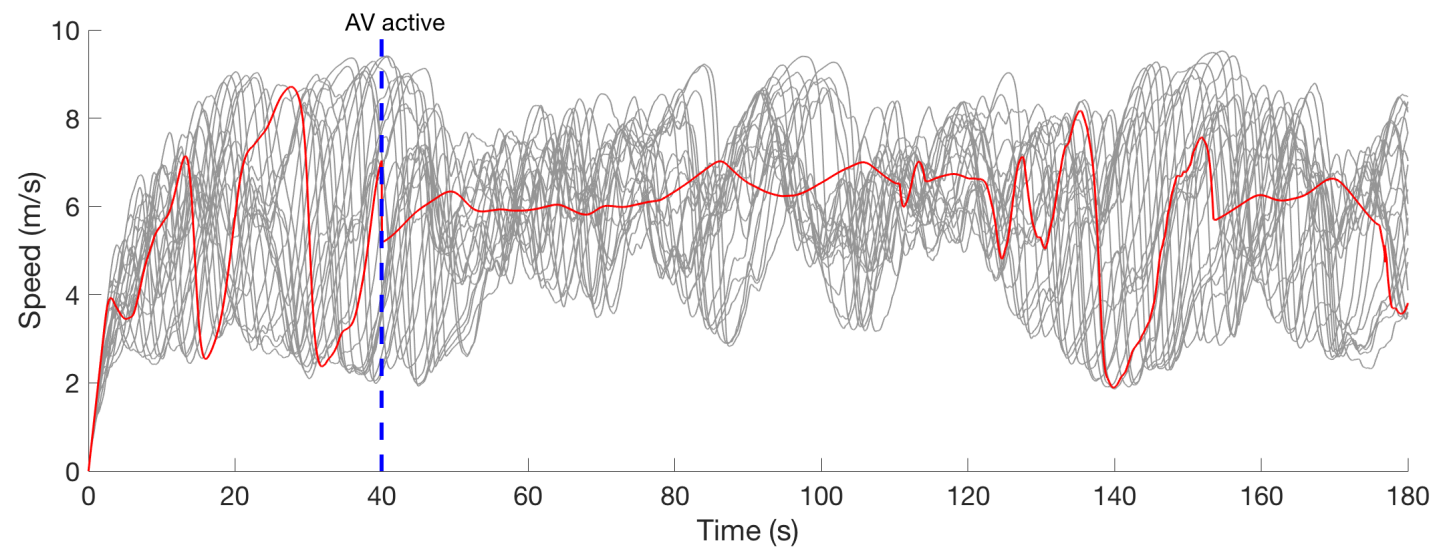

Figure 4: Velocity profiles of uncontrolled vehicles (grey) when applying the PID control to a single vehicle (red) (11). 


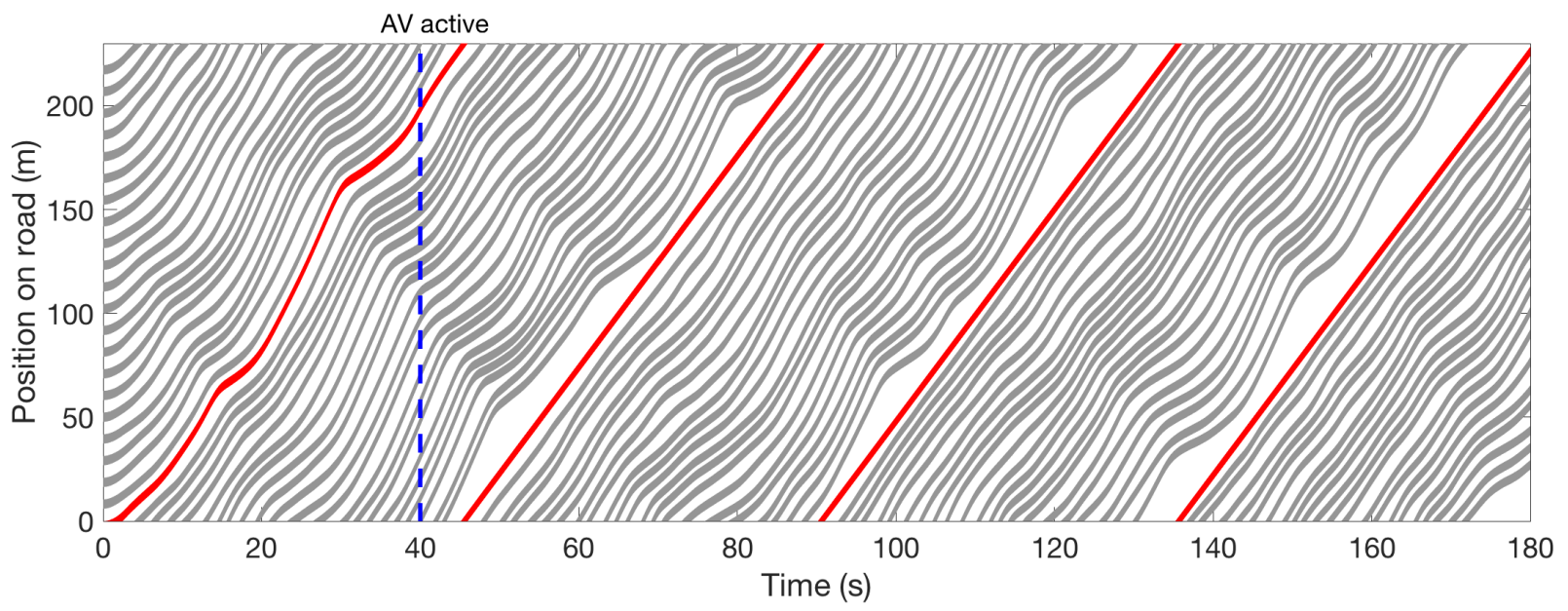

Figure 5: Vehicles trajectories when using the control (19) with (16).

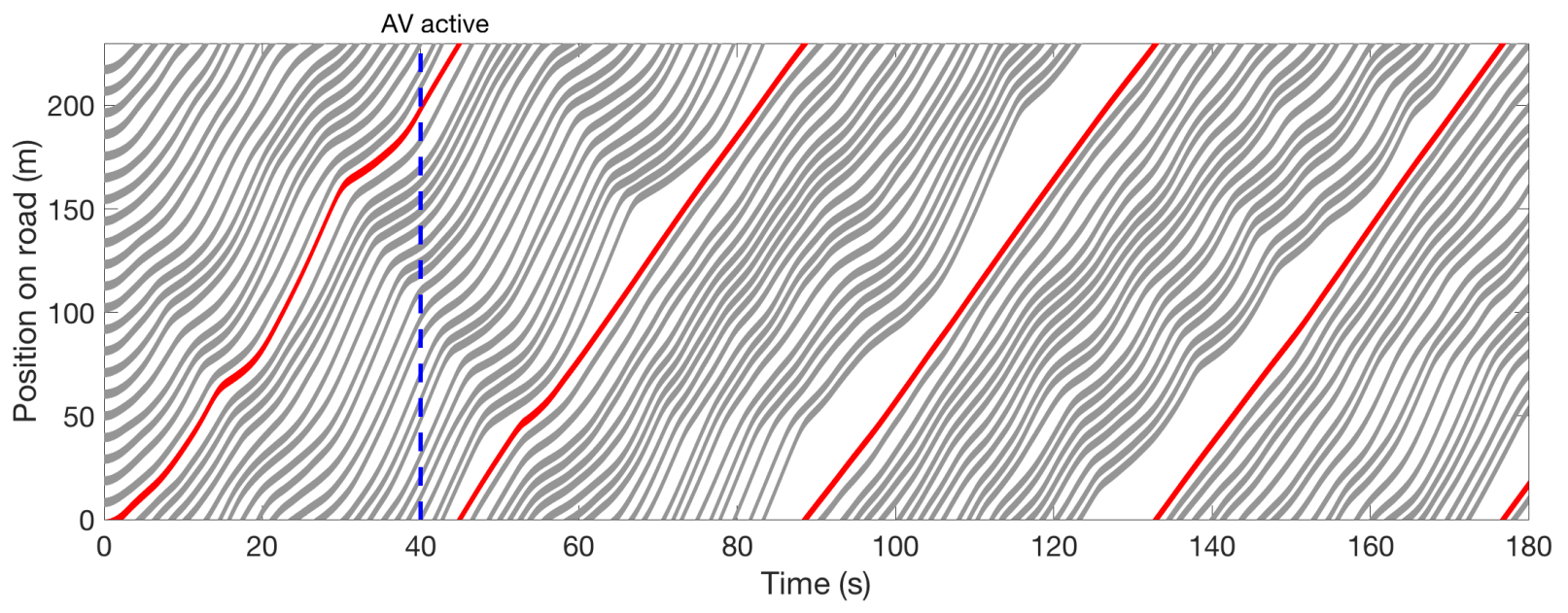

Figure 6: Vehicles trajectories when using the control (19) with (17).

The theory described in Section 4.2 is still useful because, for $j$ large enough, $v_{j} \approx c$ with $c>0$ a constant. Adding the rule (11) described in Section 4.1, the commanded velocities for (16) and (17) are updated via

$$
u_{j+1}=\beta_{j}\left(\alpha_{j} v_{k, j}^{\text {target }}+\left(1-\alpha_{j}\right) v_{j}^{\text {lead }}\right)+\left(1-\beta_{j}\right) u_{j}, \quad k \in\{1,2\} .
$$

where $v_{1, j}^{\text {target }}$ and $v_{2, j}^{\text {target }}$ are defined in (16) and (17) respectively replacing $\bar{v}$ by $\bar{v}_{j}$ defined in (18). Above, $\alpha_{j}$ and $\beta_{j}$ are constructed as explained in Section 4.1. In Figure 5 and in Figure 6, we use the commanded velocity defined in (19) with $v_{1, j}^{\text {target }}$ and $v_{2, j}^{\text {target }}$ respectively. Since the autonomous vehicle drives according to (18), a gap is created when the lead car is affected by a stop and go wave.The difference between these controls and the PID control, defined in Section 4.1, is that the autonomous vehicle never needs to catch up the lead vehicle and therefore it doesn't create another stop and go wave for the controls defined in (19). More precisely, the term $\min \left(\max \left(\frac{\Delta x-7 \mathrm{~m}}{23 \mathrm{~m}}, 0\right), 1\right)$ in (10) is not needed anymore. Moreover, in (18), we don't use that the length of the ring is equal to $L$.

The effect of the Lyapunov controllers can be seen in the velocity profiles in Figures 7, 8 which shows that the traffic is smoother when the control is active. More precisely, in Figure 7, we notice that the AV tends to have very small oscillations when the control is active. On the other side, the not controlled vehicles tend to keep some velocity oscillations, however apparently less than the PID 


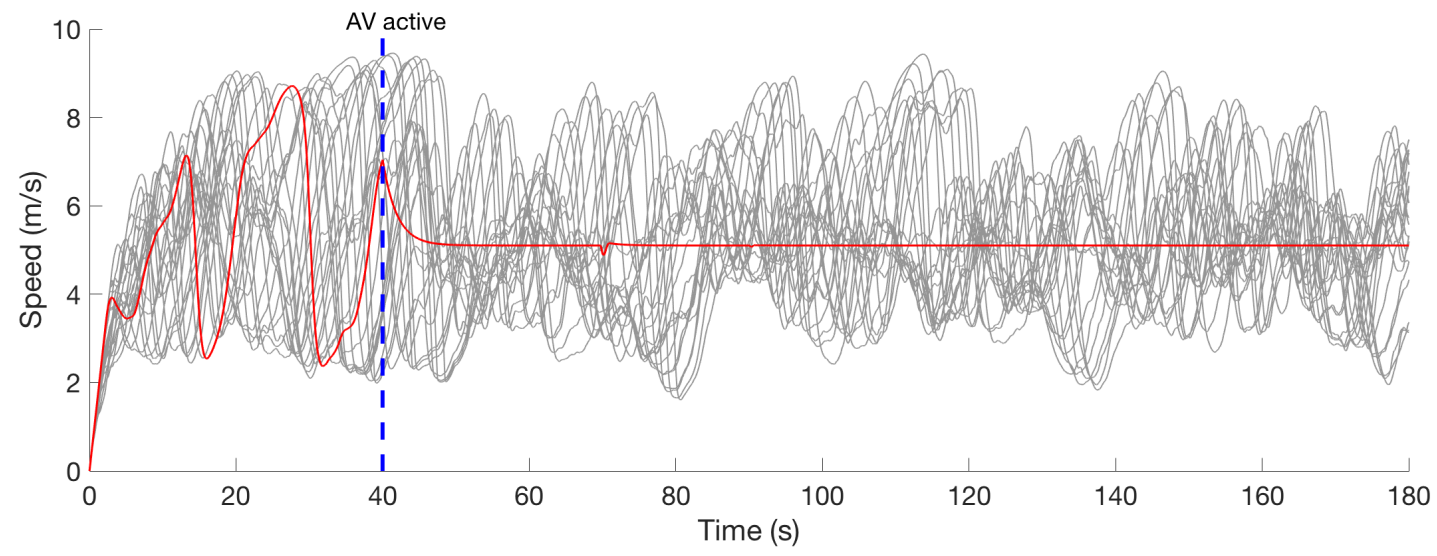

Figure 7: Simulated velocity profiles of human-piloted vehicles (grey) and autonomous vehicle (red) when using the control (19) with (16).

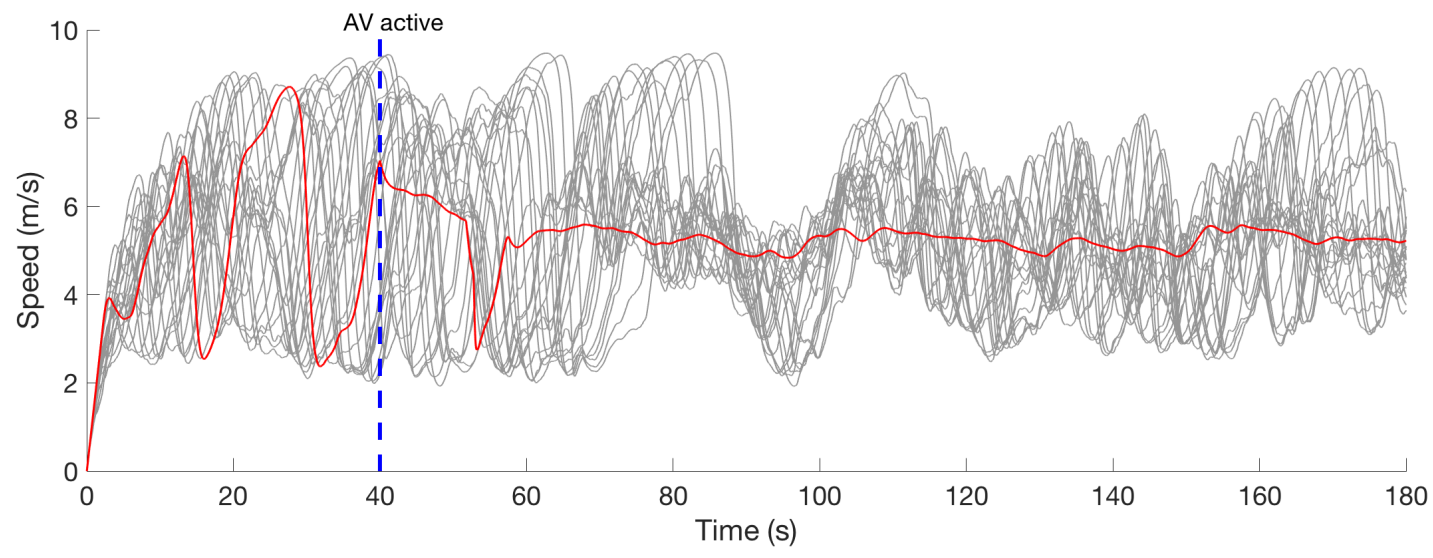

Figure 8: Simulated velocity profiles of human-piloted vehicles (grey) and autonomous vehicle (red) when using the control (19) with (17). 


\begin{tabular}{ccc}
\hline Control & $\begin{array}{c}\text { Mean fuel consumption } \\
(\ell / \mathbf{1 0 0} \mathbf{~ k m})\end{array}$ & $\begin{array}{c}\text { Standard deviation } \\
(\ell / \mathbf{1 0 0} \mathbf{~ k m})\end{array}$ \\
\hline No control & 21.93 & 0.94 \\
PID controller $(11)$ & 18.36 & 0.35 \\
$(19)$ with (16) & 18.03 & 0.46 \\
(19) with (17) & 18.13 & 0.65 \\
\hline \hline
\end{tabular}

Table 1: Simulation fuel consumption estimates over an ensemble of 10 simulation runs using the VT-Micro fuel consumption model.

controller. The interesting fact about this control is that the system, once introduced the saturation, tends to find local equilibrium and with some initial data might only dampen the stop and go waves without dissipating them. Figure 8 show a behavior pretty similar to that of the PID controller, with even stronger oscillations for the AV.

\subsection{Fuel consumption}

Fuel consumption is estimated for the simulation results using the VT-Micro fuel consumption model [45, 46]. The VT-Micro model uses polynomial regression on vehicle fuel consumption data collected at Oak Ridge National Lab to predict fuel consumption based on vehicle speed and acceleration. In simulation, per-vehicle fuel consumption averaged over an ensemble of 10 simulation runs when all traffic is under human control is $21.93 \ell / 100 \mathrm{~km}$. When the PID controller (11) is implemented on the AV in simulation and the remaining 21 vehicles are under human control, the average per-vehicle fuel consumption is reduced by $16.3 \%$ to $18.36 \ell / 100 \mathrm{~km}$. Similarly, when the controller in (19) with (16) is used on the $\mathrm{AV}$, the average per-vehicle fuel consumption is reduced by $17.8 \%$ to $18.03 \ell / 100 \mathrm{~km}$, while when the controller in (19) with (17) is used on the AV, the average per-vehicle fuel consumption is reduced by $17.3 \%$ to $18.13 \ell / 100 \mathrm{~km}$. The fuel consumption means over the 10 simulation runs conducted and corresponding standard deviations are presented in Table 1. As described in Section 4.3, the Lyapunov controllers might not always dissipate the stop-and-go waves which results in a higher standard deviation for the mean fuel consumption in Table 1 for these controls.

\section{Experimental results}

\subsection{Experimental design}

A series of experiments were conducted to validate the development of stop and go traffic waves and to demonstrate the dissipation of these waves using the designed controller. We first describe the experiment setup and its benefits and limitations. Then we present the experimental results of a low penetration rate of autonomous vehicles actively dissipating stop and go waves.

The experiments are conducted using a similar setup to the seminal works of Sugiyama, et al. [53] and Tadaki, et al. [56], which was able to isolate human driving behavior without considering other factors. These experiments showed conclusively that human driving behavior alone is sufficient to trigger stop and go waves. In this experiment, we use the same setup because it has been shown to reliably produce the kinds of stop and go waves that the controllers in this paper are designed to dampen.

While the underlying experimental setup in the work presented is similar to the setup used by Sugiyama, et al. [53], minor modifications were made to accommodate for the larger US vehicles. Just as in [53], the experiment is conducted with a total of 22 vehicles on the track. However, due to the substantially larger US vehicles, the track length was increased to $260 \mathrm{~m}$ around. While all vehicles have human-drivers in them, one of the vehicles, the University of Arizona Cognitive Autonomous Test Vehicle (CAT Vehicle) is an autonomous-capable vehicle and can be switched from being human-piloted to autonomous during the experiment.

The experiment begins with all vehicles evenly spaced and at rest. When the drivers are given a signal, they begin to drive, and human-piloted traffic conditions are observed. After some time the 


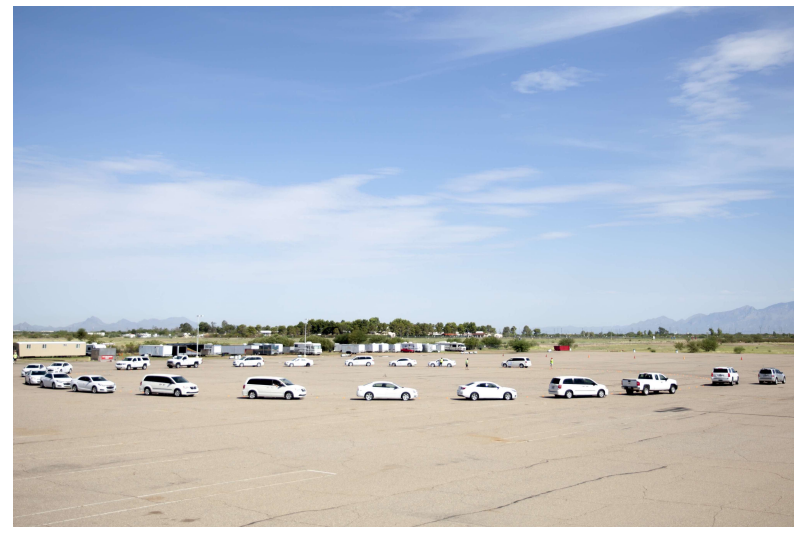

Figure 9: Vehicles on test track during the experiment.

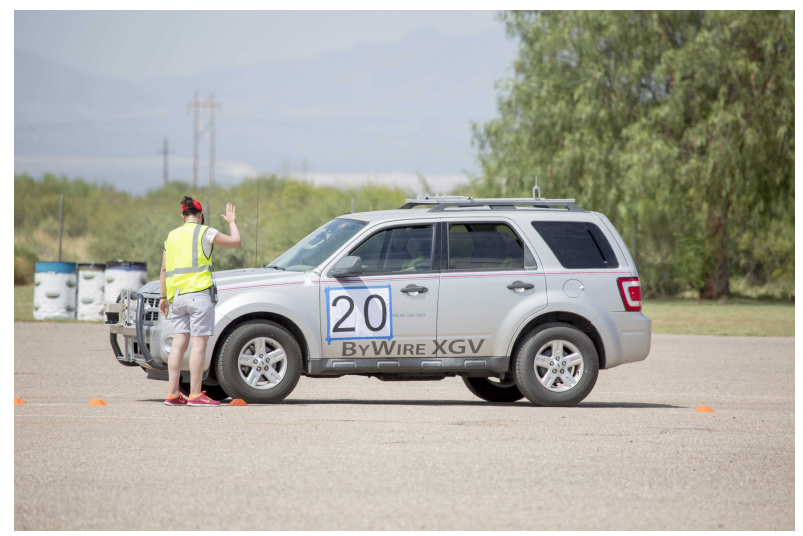

Figure 10: The CAT Vehicle, the autonomous vehicle used during the experiment.

autonomous driving capabilities of the CAT Vehicle are activated, and traffic with 21 human-drivers and one autonomous vehicle is observed.

Vehicle trajectories of each vehicle were collected using a VSN Mobile V360 panoramic video camera placed at the center of the track. The video footage recorded during the experiment was processed using image processing algorithms. More details on the image processing algorithms used can be found in the article by $\mathrm{Wu}$, et al. [59]. Additionally, vehicle performance data such as fuel consumption was recorded during the experiment using OBDLink $M X$ onboard diagnostics (OBD-II) data loggers.

The ring-road experimental design is selected because it has been shown to produce traffic instabilities that are similar to those observed in real highway traffic [53]. Furthermore, using a closed-circuit experimental test track allowed us to reproduce "infinite" traffic (where each vehicle has a vehicle in front of it and a vehicle behind it at all times) with a finite number of vehicles. However, there are some limitations that arise due to this experimental design. Since the track is only a single lane of traffic, this experimental setup cannot be used to assess the robustness of the designed algorithm to overtaking and merging.

\subsection{Experimental results}

An experiment was conducted to test the PID velocity controller in a field test. The experiment was conducted on a large flat parking lot in Tucson, AZ. The human-piloted vehicles used during this experiment were rented from the University of Arizona Motor Pool, while the AV that was used was the CAT Vehicle. An overview of the test track is seen in Figure 9 where all 22 vehicles are on the track following the experiment. The AV used to implement the traffic controller, the University of Arizona CAT Vehicle, is seen in Figure 10 being parked at the start position for the experiment.

The experiment is started with all vehicles at rest on the track and under human control. The velocity profile and vehicle trajectories for all vehicles in the experiment is presented in Figure 11 and Figure 12, respectively. Here, the CAT Vehicles speed and trajectory are plotted in red, while the speed and trajectory for the remaining vehicles are plotted in grey. After 161 seconds the small oscillations grow, and a noticeable stop and go wave develops. In the presence of this wave, vehicles fluctuate in speed between $0 \mathrm{~m} / \mathrm{s}$ and $14 \mathrm{~m} / \mathrm{s}$ as seen in Figure 11. This same stop and go wave is also seen in the vehicle trajectories in Figure 12. After 218 seconds the controller on the CAT Vehicle is activated and the traffic is under AV control. This control action is maintained until the experiment is ended after 413 seconds.

As seen in the vehicle trajectories in Figure 12, after roughly 320 seconds, one of the human-piloted vehicles introduces a small traffic wave. This wave propagates upstream until it encounters the CAT Vehicle, which is able to partially dampen, but not fully remove the wave. On the second pass around the track, the CAT Vehicle is able to fully eliminate the wave. This demonstrates that a single AV may not be sufficient to fully eliminate stop and go waves, but a low penetration rate of AVs may be sufficient to eliminate, or at least substantially dampen stop and go waves. 


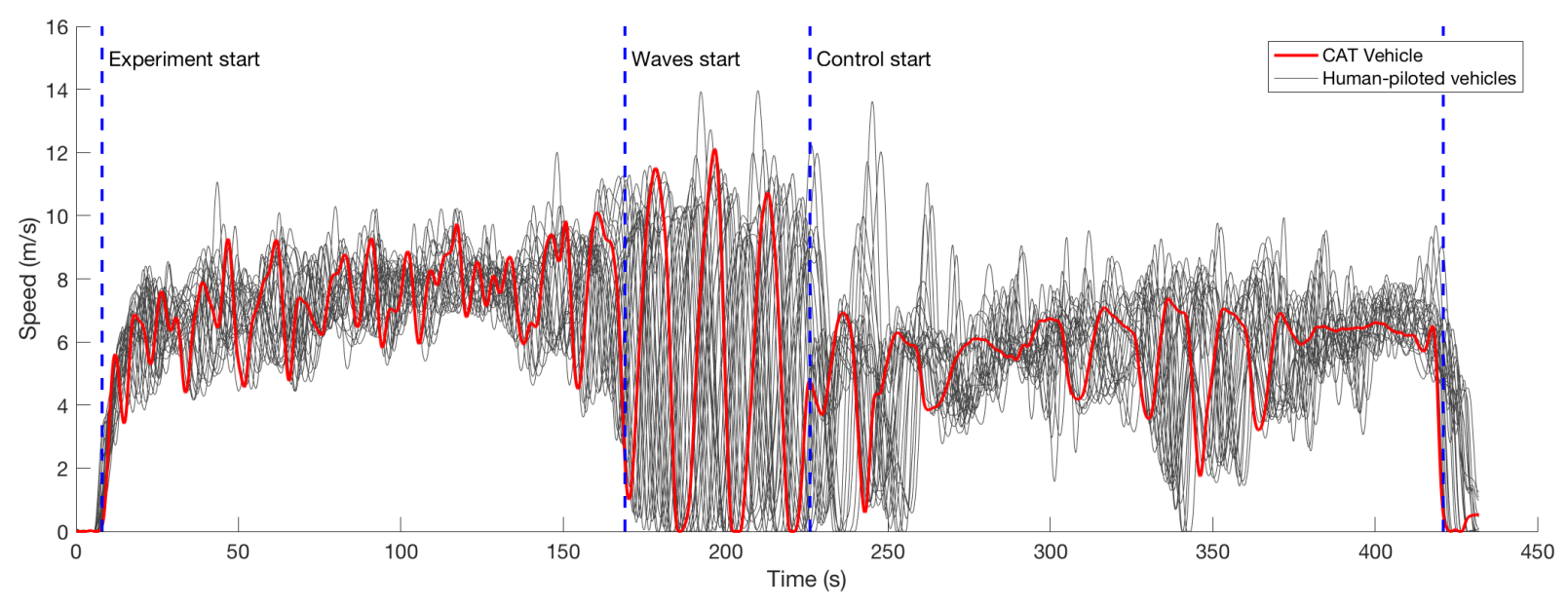

Figure 11: Velocity profile of all vehicles in the experiment. The grey velocity profiles are the human-piloted vehicles and the red profile is the CAT Vehicle. The blue vertical lines mark key times during the experiment: the start, at what point waves are clearly visible, when control of the CAT Vehicle is activated, and the end of the experiment.

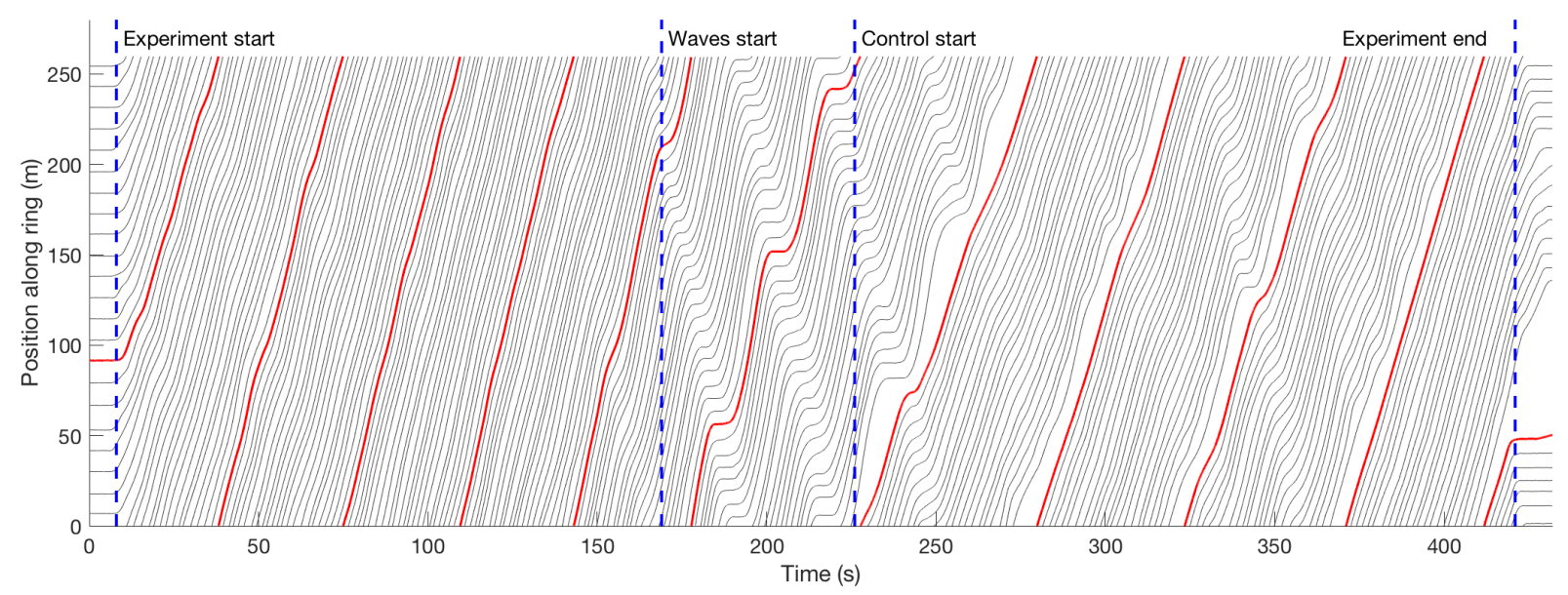

Figure 12: Trajectories of all vehicles in the experiment. The grey trajectories are the human-piloted vehicles and the red trajectory is the CAT Vehicle. The blue vertical lines mark key times during the experiment: the start, at what point waves are clearly visible, when control of the CAT Vehicle is activated, and the end of the experiment. 
The effect that the traffic controller implemented on the single AV has on the overall traffic flow (all 21 human-piloted vehicles in addition to the CAT Vehicle) is apparent in the velocity profile in Figure 11 and vehicle trajectories 12 where substantially smoother traffic is seen when the traffic is under the control of the AV. Specifically, the average velocity standard deviation between the time period where waves are present and the control period is reduced by $54.7 \%$ from $3.85 \mathrm{~m} / \mathrm{s}$ to $1.74 \mathrm{~m} / \mathrm{s}$ while not substantially effecting throughput (1755 veh/hr when waves are present and $1711 \mathrm{veh} / \mathrm{hr}$ when the AV is actively dissipating the stop and go waves, or a reduction of $2.5 \%$ ). Furthermore, since each vehicle in the flow was instrumented with an OBD-II scanner, it was possible to measure the instantaneous fuel consumption of each vehicle during the experiment. The average fuel consumption over the entire fleet when waves are present was $26.3 \ell / 100 \mathrm{~km}$, while the average fuel consumption of all vehicles when the $\mathrm{AV}$ is actively dissipating stop and go waves is reduced by $20.7 \%$ to $26.3 \ell / 100 \mathrm{~km}$. It is important to note that this reduction in fuel consumption is not only realized on the CAT Vehicle, but is the averaged quantity across all vehicles in the experiment.

This experimental result validates the theoretical and simulation-based results and demonstrates that even at a low penetration rate, AVs are capable of substantially improving traffic flow by reducing velocity standard deviation. This leads to smoother traffic and reduces fuel consumption of not only the AV, but all the vehicles in the traffic flow.

\section{Conclusion}

In this work we establish controllability results for two well known microscopic traffic flow models in the setting in which an autonomous vehicle is able to be controlled with the aim of dissipating stop and go traffic waves. Based on the controllability results, three control algorithms are developed and tested in simulation, indicating that fuel consumption reductions of up to $20 \%$ may be achieved when the flow is stabilized by the autonomous vehicle. In a first of its kind field test, we further establish for one of the proposed control algorithms that traffic wave dissipation is possible with real autonomous and human piloted vehicles, and leads to a substantial reduction in fuel consumption compared to when waves are present.

While our results show the feasibility of control of traffic via AV, especially for fuel consumption reduction, there are some limitations to our study. For instance multilane traffic was not considered and, more generally, we did not include additional challenges coming from the impact of combined complex phenomena of multilane dynamics, merges, ramps, and non-FIFO assumptions.

Looking forward, we note that the level of difficulty to prove controllability results of the combined optimal velocity follow the leader model for $n>9$ suggests alternate modeling scales, e.g., in the micromacro direction [11, 6, 28, 40], might be promising.

\section{Acknowledgement}

This research was supported by the Inria associated team "ModEling autonoMous vEhicles iN Traffic flOw " (MEMENTO). This material is based upon work supported by the National Science Foundation under Grant No. CNS-1446715 (Piccoli), CNS-1446690 (Seibold), CNS-1446435 (Sprinkle), and CNS1446702 (Work). The authors thank Hannah Pohlmann for her help in designing and tuning of the Follow-the-Leader-Bando microscopic model.

\section{References}

[1] M. Bando, H. K., A. Nakayama, A. Shibata, and Y. Sugiyama. Dynamical model of traffic congestion and numerical simulation. Phys. Rev. E, 51(2):1035-1042, 1995.

[2] N. Bellomo and C. Dogbe. On the modeling of traffic and crowds: A survey of models, speculations, and perspectives. SIAM Rev., 53(3):409-463, 2011.

[3] B. Besselink and K. H. Johansson. String stability and a delay-based spacing policy for vehicle platoons subject to disturbances. IEEE Transactions on Automatic Control, 2017. 
[4] A. Bressan and B. Piccoli. Introduction to the Mathematical Theory of Control, volume 2 of AIMS Series on Applied Mathematics. American Institute of Mathematical Sciences (AIMS), Springfield, MO, 2007.

[5] M. Buehler, K. Iagnemma, and S. Singh. The DARPA urban challenge: autonomous vehicles in city traffic, volume 56. Springer, 2009.

[6] C. Chalons, M. L. Delle Monache, and P. Goatin. A conservative scheme for non-classical solutions to a strongly coupled pde-ode problem. Interfaces and Free Boundaries, 19(4):553-571, 2017.

[7] L. Chong, M. Abbas, and A. Medina. Simulation of driver behavior with agent-based backpropagation neural network. Transportation Research Record: Journal of the Transportation Research Board, 2249:44-51, 2011.

[8] J.-M. Coron. Control and nonlinearity. Number 136. American Mathematical Soc., 2007.

[9] S. Darbha and K. Rajagopal. Intelligent cruise control systems and traffic flow stability. Transportation Research Part C: Emerging Technologies, 7(6):329 - 352, 1999.

[10] L. Davis. Effect of adaptive cruise control systems on traffic flow. Physical Review E, 69(6):066110, 2004.

[11] M. L. Delle Monache and P. Goatin. Scalar conservation laws with moving constraints arising in traffic flow modeling: an existence result. Journal of Differential equations, 257(11):4015-4029, 2014.

[12] R. E. Fenton and R. J. Mayhan. Automated highway studies at the Ohio State University-an overview. IEEE transactions on Vehicular Technology, 40(1):100-113, 1991.

[13] M. Garavello, K. Han, and B. Piccoli. Models for vehicular traffic on networks. American Institute of Mathematical Sciences, 2016.

[14] G. I. Ge, S. S. Avedisov, C. R. He, W. B. Qin, M. Sadeghpour, and G. Orosz. Experimental validation of connected automated vehicle design among human-driven vehicles. Transportation research part C: emerging technologies, 91:335-352, 2018.

[15] G. Gomes and R. Horowitz. Optimal freeway ramp metering using the asymmetric cell transmission model. Transportation Research Part C: Emerging Technologies, 14(4):244-262, 2006.

[16] M. Guériau, R. Billot, N.-E. El Faouzi, J. Monteil, F. Armetta, and S. Hassas. How to assess the benefits of connected vehicles? a simulation framework for the design of cooperative traffic management strategies. Transportation Research Part C: Emerging Technologies, 67:266-279, 2016.

[17] Y. Han, A. Hegyi, Y. Yuan, S. Hoogendoorn, M. Papageorgiou, and C. Roncoli. Resolving freeway jam waves by discrete first-order model-based predictive control of variable speed limits. Transportation Research Part C: Emerging Technologies, 77:405-420, 2017.

[18] A. Hegyi, B. De Schutter, and H. Hellendoorn. Model predictive control for optimal coordination of ramp metering and variable speed limits. Transportation Research Part C: Emerging Technologies, 13(3):185-209, 2005.

[19] A. Hegyi, B. De Schutter, and J. Hellendoorn. Optimal coordination of variable speed limits to suppress shock waves. IEEE Transactions on Intelligent Transportation Systems, 6(1):102-112, 2005.

[20] A. Hegyi, S. Hoogendoorn, M. Schreuder, H. Stoelhorst, and F. Viti. SPECIALIST: A dynamic speed limit control algorithm based on shock wave theory. In Proceedings of the IEEE Conference on Intelligent Transportation Systems, pages 827-832. IEEE, 2008.

[21] D. Helbing. Traffic and related self-driven many-particle systems. Reviews of Modern Physics, 73:1067-1141, 2001.

[22] J. Hongfei, J. Zhicai, and N. Anning. Develop a car-following model using data collected by" five-wheel system". In Proceedings of the IEEE Conference on Intelligent Transportation Systems, volume 1, pages 346-351. IEEE, 2003.

[23] P. Ioannou, Z. Xu, S. Eckert, D. Clemons, and T. Sieja. Intelligent cruise control: theory and experiment. In Proceedings of the 32nd IEEE Conference on Decision and Control, pages 18851890. IEEE, 1993. 
[24] V. Jurdjevic and J. P. Quinn. Controllability and stability. J. Differential Equations, 28(3):381-389, 1978.

[25] A. Khodayari, A. Ghaffari, R. Kazemi, and R. Braunstingl. A modified car-following model based on a neural network model of the human driver effects. IEEE Transactions on Systems, Man, and Cybernetics-Part A: Systems and Humans, 42(6):1440-1449, 2012.

[26] F. Knorr, D. Baselt, M. Schreckenberg, and M. Mauve. Reducing traffic jams via VANETs. IEEE Transactions on Vehicular Technology, 61(8):3490-3498, 2012.

[27] K. Konishi, H. Kokame, and K. Hirata. Decentralized delayed-feedback control of an optimal velocity traffic model. The European Physical Journal B-Condensed Matter and Complex Systems, 15(4):715722,2000 .

[28] C. Lattanzio, A. Maurizi, and B. Piccoli. Moving bottlenecks in car traffic flow: A PDE-ODE coupled model. SIAM J. Math. Anal., 43:50-67, 2011.

[29] W. Levine and M. Athans. On the optimal error regulation of a string of moving vehicles. IEEE Transactions on Automatic Control, 11(3):355-361, 1966.

[30] X.-Y. Lu, Z. Qiu, P. Varaiya, R. Horowitz, and S. E. Shladover. Combining variable speed limits with ramp metering for freeway traffic control. In Proceedings of the American Control Conference, pages 2266-2271, 2010.

[31] X.-Y. Lu, S. E. Shladover, I. Jawad, R. Jagannathan, and T. Phillips. Novel algorithm for variable speed limits and advisories for a freeway corridor with multiple bottlenecks. Transportation Research Record: Journal of the Transportation Research Board, 2489:86-96, 2015.

[32] J. Ma, X. Li, S. E. Shladover, H. A. Rakha, X.-Y. Lu, R. Jagannathan, and D. J. Dailey. Freeway speed harmonization. IEEE Transactions on Intelligent Vehicles, 1(1):78-89, 2016.

[33] V. Milanés, S. E. Shladover, J. Spring, C. Nowakowski, H. Kawazoe, and M. Nakamura. Cooperative adaptive cruise control in real traffic situations. IEEE Transactions on Intelligent Transportation Systems, 15(1):296-305, 2014.

[34] A. Nakayama, Y. Sugiyama, and K. Hasebe. Effect of looking at the car that follows in an optimal velocity model of traffic flow. Physical Review E, 65(1):016112, 2001.

[35] A. Nissan and H. N. Koutsopoulos. Evaluation of the impact of advisory variable speed limits on motorway capacity and level of service. Procedia-Social and Behavioral Sciences, 16:100-109, 2011.

[36] G. Orosz, R. E. Wilson, and G. Stépán. Traffic jams: dynamics and control, 2010.

[37] M. Papageorgiou, H. Hadj-Salem, and J.-M. Blosseville. ALINEA: A local feedback control law for on-ramp metering. Transportation Research Record: Journal of the Transportation Research Board, 1320:58-67, 1991.

[38] M. Papageorgiou and A. Kotsialos. Freeway ramp metering: An overview. IEEE Transactions on Intelligent Transportation Systems, 3(4):271-281, 2002.

[39] I. Papamichail, K. Kampitaki, M. Papageorgiou, and A. Messmer. Integrated ramp metering and variable speed limit control of motorway traffic flow. IFAC Proceedings Volumes, 41(2):14084-14089, 2008.

[40] G. Piacentini, P. Goatin, and A. Ferrara. Traffic control via moving bottleneck of coordinated vehicles. In 15th IFAC symposium on control in transportation systems, 2018.

[41] B. Piccoli and A. Tosin. Vehicular Traffic: A Review of Continuum Mathematical Models, pages 1748-1770. Springer New York, New York, NY, 2011.

[42] L. A. Pipes. An operational analysis of traffic dynamics. Journal of applied physics, 24(3):274-281, 1953.

[43] A. Popov, A. Hegyi, R. Babuška, and H. Werner. Distributed controller design approach to dynamic speed limit control against shockwaves on freeways. Transportation Research Record: Journal of the Transportation Research Board, 2086:93-99, 2008.

[44] R. Rajamani, S. B. Choi, J. K. Law, B. K.and Hedrick, R. Prohaska, and P. Kretz. Design and experimental implementation of control for a platoon of automated vehicles. AMSE Journal of Dynamic Systems, Measurement, and Control, 122(3):470-476, 1998. 
[45] H. Rakha, K. Ahn, and A. Trani. Comparison of mobile5a, mobile6, vt-micro, and cmem models for estimating hot-stabilized light-duty gasoline vehicle emissions. Canadian Journal of Civil Engineering, 30(6):1010-1021, 2003.

[46] H. Rakha, K. Ahn, and A. Trani. Development of VT-Micro model for estimating hot stabilized light duty vehicle and truck emissions. Transportation Research Part D: Transport and Environment, 9(1):49-74, 2004.

[47] A. Reuschel. Vehicle movements in a platoon. Oesterreichisches Ingenieur-Archir, 4:193-215, 1950.

[48] A. Reuschel. Vehicle movements in a platoon with uniform acceleration or deceleration of the lead vehicle. Zeitschrift des Oesterreichischen Ingenieur-und Architekten-Vereines, 95:50-62, 1950.

[49] SAE. Taxonomy and definitions for terms related to on-road motor vehicle automated driving systems. Technical report, 2014.

[50] S. E. Shladover. Review of the state of development of advanced vehicle control systems (avcs). Vehicle System Dynamics, 24(6-7):551-595, 1995.

[51] S. Smulders. Control of freeway traffic flow by variable speed signs. Transportation Research Part B: Methodological, 24(2):111-132, 1990.

[52] Y. Sugiyama, M. Fukui, M. Kikuchi, K. Hasebe, A. Nakayama, K. Nishinari, S. Tadaki, and S. Yukawa. Traffic jams without bottlenecks - experimental evidence for the physical mechanism of the formation of a jam. New Journal of Physics, 10(3):033001, 2008.

[53] Y. Sugiyama, M. Fukui, M. Kikuchi, K. Hasebe, A. Nakayama, K. Nishinari, S.-i. Tadaki, and S. Yukawa. Traffic jams without bottlenecks-experimental evidence for the physical mechanism of the formation of a jam. New Journal of Physics, 10(3):033001, 2008.

[54] D. Swaroop and J. Hedrick. String stability of interconnected systems. IEEE transactions on automatic control, 41(3):349-357, 1996.

[55] S. Tadaki, M. Kikuchi, M. Fukui, A. Nakayama, K. Nishinari, A. Shibata, Y. Sugiyama, T. Yosida, and S. Yukawa. Phase transition in traffic jam experiment on a circuit. New Journal of Physics, 15:103034, 2013.

[56] S.-i. Tadaki, M. Kikuchi, M. Fukui, A. Nakayama, K. Nishinari, A. Shibata, Y. Sugiyama, T. Yosida, and S. Yukawa. Phase transition in traffic jam experiment on a circuit. New Journal of Physics, 15(10):103034, 2013.

[57] A. Talebpour and H. S. Mahmassani. Influence of connected and autonomous vehicles on traffic flow stability and throughput. Transportation Research Part C: Emerging Technologies, 71:143-163, 2016.

[58] M. Wang, W. Daamen, S. P. Hoogendoorn, and B. van Arem. Cooperative car-following control: Distributed algorithm and impact on moving jam features. IEEE Transactions on Intelligent Transportation Systems, 17(5):1459-1471, 2016.

[59] F. Wu, R. Stern, S. Cui, M. L. Delle Monache, R. Bhadani, M. Bunting, M. Churchill, N. Hamilton, R. Haulcy, H. Pohlmann, B. Piccoli, B. Seibold, J. Sprinkle, and D. B. Work. Tracking vehicle trajectories and fuel rates in oscillatory traffic. Transportation Research Part C: Emerging Technologies, under review, 2017.

[60] M. Zhou, X. Qu, and X. Li. A recurrent neural network based microscopic car following model to predict traffic oscillation. Transportation Research Part C: Emerging Technologies, 84:245-264, 2017.

\section{Appendix}

The linearization of system (8) at the equilibrium point $\left(Y^{*}, 0\right) \in \mathbb{R}^{2 n}$ is described by

$$
\dot{Y}=A Y+B u
$$


where

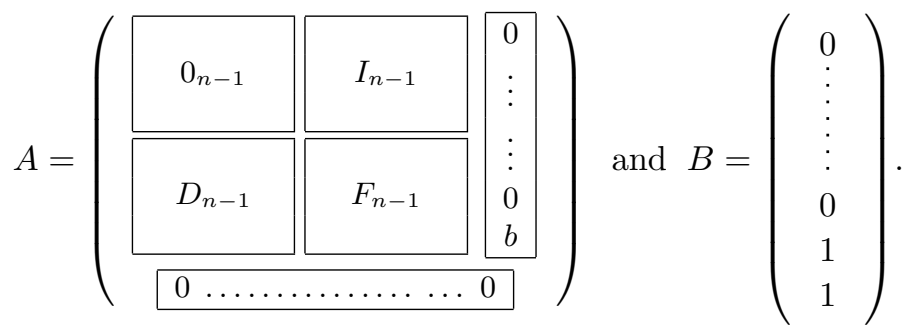

For $n \in \mathbb{N}$ define the matrices $\left(D_{n-1}, F_{n-1}\right) \in \mathcal{M}_{2 n-1}(\mathbb{R})^{2}$ by:

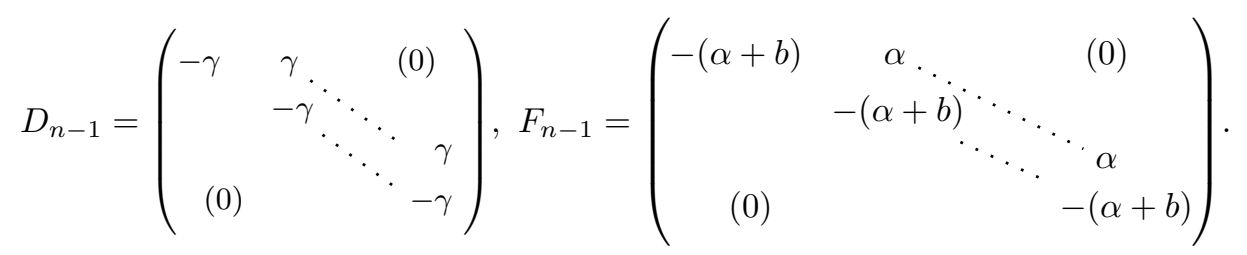

with $\gamma=b V^{\prime}\left(y^{*}+d\right)$ and $\alpha=\frac{a}{\left(y^{*}+d\right)^{2}}$. Moreover, denote by $0_{n-1} \in \mathcal{M}_{n-1}(\mathbb{R})$ and $I_{n-1} \in \mathcal{M}_{n-1}(\mathbb{R})$ the zero matrix and the identity matrix respectively. Let $k \geqslant 1$. From (21), there exist $\left(A_{i, k}\right)_{i \in\{1, \cdots, 4\}} \in$ $\mathcal{M}_{n-1}(\mathbb{R})$ and $\left(C_{i, k}\right)_{i \in\{1,2\}} \in \mathcal{M}_{n-1,1}(\mathbb{R})$ such that,

$$
A^{k}=\left[\begin{array}{ccc}
A_{1, k} & A_{2, k} & C_{1, k} \\
A_{3, k} & A_{4, k} & C_{2, k} \\
0_{1, n-1} & 0_{1, n-1} & 0
\end{array}\right]
$$

and for every $k \geqslant 1$ we have

$$
\left\{\begin{array}{l}
A_{4, k+1}=D_{n-1} A_{2, k}+F_{n-1} A_{4, k} \\
A_{2, k+1}=A_{4, k} \\
C_{2, k+1}=D_{n-1} C_{1, k}+F_{n-1} C_{2, k} \\
C_{1, k+1}=C_{2, k}
\end{array}\right.
$$

In particular, for every $k \geqslant 2$,

$$
\left\{\begin{array}{l}
A_{4, k+1}=D_{n-1} A_{4, k-1}+F_{n-1} A_{4, k} \\
A_{2, k+1}=D_{n-1} A_{2, k-1}+F_{n-1} A_{2, k} \\
C_{2, k+1}=D_{n-1} C_{2, k-1}+F_{n-1} C_{2, k} \\
C_{2, k}=D_{n-1} C_{1, k-1}+F_{n-1} C_{1, k}
\end{array}\right.
$$

Combining (23) with (24) and using that, for every $k \geqslant 1, A^{k} B(2 n-1)=0$, we conclude that, for every $k \geqslant 2$,

$$
A^{k+1} B=\mathcal{D} A^{k-1} B+\mathcal{F} A^{k} B
$$

with

$$
\mathcal{D}=\left[\begin{array}{ccc}
D_{n-1} & 0_{n-1} & 0 \\
0_{n-1} & D_{n-1} & 0 \\
0_{1, n-1} & 0_{1, n-1} & 0
\end{array}\right] \quad \text { and } \quad \mathcal{F}=\left[\begin{array}{ccc}
F_{n-1} & 0_{n-1} & 0 \\
0_{n-1} & F_{n-1} & 0 \\
0_{1, n-1} & 0_{1, n-1} & 0
\end{array}\right]
$$

Moreover, we have

$$
A^{2} B=X+\mathcal{F}_{2 n-1} A B \quad \text { with } A B=\left(\begin{array}{l}
0_{n-2,1} \\
1 \\
0_{n-3,1} \\
\alpha \\
-\alpha \\
0
\end{array}\right) \text { and } X=\left(\begin{array}{l}
0_{n-2,1} \\
b \\
0_{n-3,1} \\
\gamma \\
-\gamma \\
0
\end{array}\right)
$$


Proof of Theorem 3. If $b=0$ then $\gamma=0$. From (25) and (27), for every $k \geqslant 2$,

$$
\left\{\begin{array}{l}
A^{k+1}=\mathcal{F} A^{k} B \\
A^{2} B=\mathcal{F} A B
\end{array}\right.
$$

Thus, the Kalman controllability matrix (6) satisfies

$$
\operatorname{rank}(K(A, B))=\operatorname{rank}\left(B, A B, \mathcal{F} A B, \cdots, \mathcal{F}^{2 n-4} A B\right) .
$$

By Cayley-Hamilton Theorem, there exists $\left(\alpha_{0}, \cdots, \alpha_{n-2}\right) \in \mathbb{R}^{n-1}$ such that $F_{n-1}^{n-1}=\sum_{i=0}^{n-2} \alpha_{i} F_{n-1}^{i}$. From (26), we conclude that

$$
\operatorname{rank}(K(A, B))=\operatorname{rank}\left(B, A B, \mathcal{F} A B, \cdots, \mathcal{F}^{n-2} A B\right)
$$

Using the expression of $A B$ given in (27) and the equality $\mathcal{F}^{k}=\left[\begin{array}{ccc}F_{n-1}^{k} & 0_{n-1} & 0 \\ 0_{n-1} & F_{n-1}^{k} & 0 \\ 0_{1, n-1} & 0_{1, n-1} & 0\end{array}\right]$, by straightforward computations, we have

$$
\operatorname{rank}\left(B, A B, \mathcal{F} A B, \cdots, \mathcal{F}^{n-2} A B\right)=n
$$

whence the conclusion.

Proof of Theorem 4. Let's prove by induction that, for every $k \geqslant 1$, there exist $\left(\lambda_{i}\right)_{i=1, \cdots, 2 k}$ and $\left(\mu_{i}\right)_{i=1, \cdots, 2 k+1}$ such that

$$
\left\{\begin{array}{l}
A^{2 k+1} B=\sum_{i=1}^{2 k} \lambda_{i} A^{i} B+\mathcal{D}^{k}(A B) \\
A^{2 k+2} B=\sum_{i=1}^{2 \bar{k}+1} \mu_{i} A^{i} B+\mathcal{D}^{k}\left(A^{2} B\right)
\end{array} .\right.
$$

Since $a=0$, we have $F_{n-1}=-b I_{n-1}$. Using $(25), A^{3} B=\mathcal{D} A B-b A^{2} B$ and $A^{4} B=\mathcal{D} A^{2} B-b A^{3} B$. Thus, $\left(P_{k}\right)$ holds for $k=1$. Assuming that $\left(P_{k}\right)$ holds for $k=p$. From (25), we have

$$
\left\{\begin{array}{l}
A^{2 p+3}=\mathcal{D} A^{2 p+1}-b A^{2 p+2} B \\
A^{2 p+4}=\mathcal{D} A^{2 p+2}-b A^{2 p+3} B
\end{array} \quad \text { and } \quad \mathcal{D} A^{i} B=A^{i+2} B+b A^{i+1} B, i \geqslant 1 .\right.
$$

Using (28) and $\left(P_{k}\right)$ for $k=p$, we conclude that $\left(P_{k}\right)$ holds for $k=p+1$.

The equality $\left(P_{k}\right)$ for $k=n-2$ gives

$$
\begin{aligned}
\operatorname{rank}(K(A, B)) & =\left(B, A B, \cdots, A^{2 n-3}, \mathcal{D}^{n-2} A^{2} B\right) \\
& =\left(B, A B, \cdots, A^{2 n-4}, \mathcal{D}^{n-2} A B, \mathcal{D}^{n-2} A^{2} B\right) \\
& =\left(B, A B, A^{2} B, \mathcal{D} A B, \mathcal{D} A^{2} B, \cdots, \mathcal{D}^{n-2} A B, \mathcal{D}^{n-2} A^{2} B\right)
\end{aligned}
$$

Since $A B$ and $A^{2} B$ are linearly independent and $\mathcal{D}^{k}=\left[\begin{array}{ccc}D_{n-1}^{k} & 0_{n-1} & 0 \\ 0_{n-1} & D_{n-1}^{k} & 0 \\ 0_{1, n-1} & 0_{1, n-1} & 0\end{array}\right]$, by straightforward computations, we have

$$
\operatorname{rank}\left(B, A B, A^{2} B, \mathcal{D} A B, \mathcal{D} A^{2} B, \cdots, \mathcal{D}^{n-2} A B, \mathcal{D}^{n-2} A^{2} B\right)=2 n-1 .
$$

Thus, the linearization of system $(8)$ at the equilibrium point $\left(Y^{*}, 0\right)$ is controllable. Using [8, Theorem 3.8], Theorem 4 is proved.

Proof of Theorem 5. Using using the symbolic mathematics software Maple [https://www . maplesoft. com/products/Maple/], we establish that for every $3 \leqslant n \leqslant 9$,

$$
\operatorname{Det}(K(A, B))=\gamma^{\frac{n^{2}-3 n+2}{2}} b^{\frac{n^{2}-n}{2}}\left(\alpha-\frac{\gamma}{b}\right)^{\frac{n^{2}-n}{2}} .
$$

Thus, for every $\alpha \neq \frac{\gamma}{b}, \gamma \neq 0, b \neq 0$, the linearization of system (8) at the equilibrium point $\left(Y^{*}, 0\right)$ is controllable, whence the conclusion of Theorem 5 by using [8, Theorem 3.8]. 